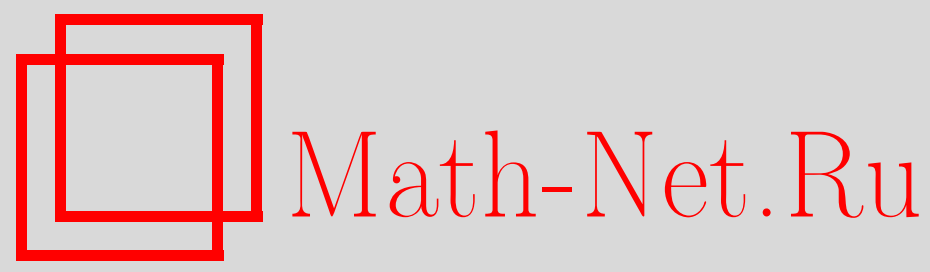

Н. А. Шананин, О частичной квазианалитичности обобщенных решений слабо нелинейных дифференциальных уравнений со взвешенными производными, Матем. заметки, 2000, том 68, выпуск 4, 608-619

DOI: https://doi.org/10.4213/mzm981

Использование Общероссийского математического портала Math-Net.Ru подразумевает, что вы прочитали и согласны с пользовательским соглашением http://www . mathnet.ru/rus/agreement

Параметры загрузки:

IP : 54.224 .135 .184

26 апреля 2023 г., $17: 48: 20$

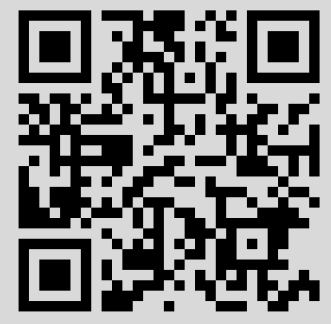




\section{О ЧАСТИЧНОЙ КВАЗИАНАЛИТИЧНОСТИ ОБОБЩЕННЫХ РЕШЕНИЙ СЛАБО НЕЛИНЕЙНЫХ ДИФФЕРЕНЦИАЛЬНЫХ УРАВНЕНИЙ СО ВЗВЕШЕННЫМИ ПРОИЗВОДНЫМИ}

\section{Н. А. Шананин}

Получены достаточные условия, при которых множество обобщенных решений слабо нелинейного дифференциального уравнения со взвешенными производными образует квазианалитический класс относительно части переменных. Рассматриваемый класс уравнений содержит ряд уравнений, описывающих распространение нелинейных волн.

Библиограффия: 6 названий.

Известно, что при выполнении определенных условий на старшую часть эллиптического дифференциального уравнения с неаналитическими коэффициентами множество обобщенных решений этого уравнения порождает квазианалитический класс в следуюшем смысле: из совпадения двух решений в окрестности какой-либо точки области следует их тождественность во всей области. Для ряда слабо нелинейных дифференциальных уравнений со взвешенными производными имеет место близкий эффект продолжения обобщенных решений по той части переменньх, дифференцированиям по которым присвоен минимальньй вес. В статье указаны достаточные условия, при которых множество достаточно гладких обобщенных решений слабо нелинейного дифференциального уравнения со взвешенными производными образует квазианалитический класс по части переменных. Класс уравнений включает ряд нелинейных уравнений, описываюших волновые процессы.

Теорема 1 содержит основной результат статьи. Теорема 2 , доказательство которой вынесено в дополнение, позволяет при определенных условиях приводить нелинейную часть слабо нелинейного уравнения к виду, рассмотренному в теореме 1 . Приложениям теоремы 1 к конкретным уравнениям посвящен п. 2 .

1. Основные результаты. Пусть в открытом множестве $\Omega \subset \mathbb{R}^{n}$ определен линейньй дифференциальный оператор со взвешенными производными

$$
P(x, D)=\sum_{\langle\varrho, \alpha\rangle \leqslant m} a_{\alpha}(x) D^{\alpha}, \quad D_{j}=\frac{1}{i} \frac{\partial}{\partial x_{j}}, \quad j=1,2, \ldots, n,
$$

Работа выполнена при поддержке Российского фонда фундаментальных исследований, грант № 99-01-01139. 
где $\alpha=\left(\alpha_{1}, \alpha_{2}, \ldots, \alpha_{n}\right)$ - целочисленный неотрицательньй мультииндекс, веса $\varrho=$ $\left(\varrho_{1}, \varrho_{2}, \ldots, \varrho_{n}\right)$ - натуральные числа, $\langle\varrho, \alpha\rangle-$ взвешенный порядок производной $D^{\alpha}$ : $\langle\varrho, \alpha\rangle=\varrho_{1} \alpha_{1}+\varrho_{2} \alpha_{2}+\cdots+\varrho_{n} \alpha_{n}$. Обозначим через $\mu$ минимальньй вес: $\mu=\min \varrho_{j}$. Будем предполагать, что коэффициенты $a_{\alpha}(x) \in L_{\infty, \text { loc }}(\Omega)$ при $\langle\varrho, \alpha\rangle \leqslant m-\mu$ и $a_{\alpha}(x) \in$ $C^{\infty}(\Omega)$ при $m-\mu<\langle\varrho, \alpha\rangle \leqslant m$, причем по крайней мере один из коэффициентов $a_{\alpha}(x)$ при $\langle\varrho, \alpha\rangle=m$ отличен от тождественного нуля. В этом случае число $m$ назьвается взвешенным порядком оператора.

Полный символ дифференциального оператора (1) является суммой квазиоднородных по $\xi \in \mathbb{R}^{n}$ слагаемых

$$
p_{k}(x, \xi)=\sum_{\langle\varrho, \alpha\rangle=k} a_{\alpha}(x) \xi^{\alpha}
$$

степеней квазиоднородности $k=0,1, \ldots, m$, соответственно $(x, \xi) \in \Omega \times \mathbb{R}^{n}$. Символ максимальной степени $p_{m}(x, \xi)$ называется взвешенным главным символом оператоpa $P(x, D)$.

Координаты $x=\left(x_{1}, \ldots, x_{n}\right)$ разделим на две части $x^{\prime}=\left\{x_{j} \mid \varrho_{j}=\mu\right\}$ и $x^{\prime \prime}=\left\{x_{j} \mid\right.$ $\left.\varrho_{j}>\mu\right\}$ соответственно, $\xi=\left(\xi^{\prime}, \xi^{\prime \prime}\right)$. Оператор $P$ называется $\xi^{\prime}$-квазиэллиптическим в точке $x \in \Omega$, если из равенств $p_{m}(x, \xi)=0$ и $\xi^{\prime \prime}=0$ следует $\xi^{\prime}=0$. Оператор $P$ назьвается $\xi^{\prime}$-квазиэллиптическим в $\Omega$, если он является $\xi^{\prime}$-квазиэллиптическим во всех точках $\Omega$. Гиперповерхность $S=\{x \in U \mid \varphi(x)=0\}$, где $U$ - область в $\Omega, \varphi(x)-$ вешественная $C^{\infty}$-функция и $d \varphi \neq 0$ в $U$, назьвается нехарактеристической в точке $x^{0} \in S$ для оператора $P(x, D)$, если $p_{m}\left(x^{0}, \varphi_{x^{\prime}}\left(x^{0}\right), 0\right) \neq 0$. Очевидно, если оператор $P$ является $\xi^{\prime}$-квазиэллиптическим в $x^{0}$, то для того чтобы $S$ была нехарактеристической в $x^{0}$, необходимо и достаточно, чтобы $\varphi_{x^{\prime}}\left(x^{0}\right) \neq 0$.

Пусть оператор $P$ является $\xi^{\prime}$-квазиэллиптическим в $\Omega$. Тогда для любых $x^{0} \in \Omega$, $\eta^{\prime 0} \neq 0$ и $\xi^{0}=\left(\xi^{\prime 0}, \xi^{\prime \prime 0}\right)$ многочлен $p_{m}\left(x^{0}, \xi^{\prime 0}+z{\eta^{\prime}}^{0}, \xi^{\prime \prime 0}\right)$ относительно $z \in \mathbb{C}$ имеет степень $m / \mu$.

Предположим, что

I) оператор $P$ является $\xi^{\prime}$-квазиэллиптическим в $\Omega$ и для любых точки $x^{0} \in \Omega$ и неколлинеарных векторов $\left(\eta^{\prime 0}, 0\right)$ и $\left(\xi^{\prime 0}, \xi^{\prime \prime 0}\right)$ многочлен $p_{m}\left(x^{0}, \xi^{\prime 0}+z \eta^{\prime 0}, \xi^{\prime \prime 0}\right)$ имеет $m / \mu$ простых корней.

С оператором $P(x, D)$ свяжем пучок стариих символов

$$
\mathscr{H}_{P}(x, \xi, h)=\sum_{k=0}^{\mu-1} h^{k} p_{m-k}(x, \xi), \quad h \in \mathbb{R} .
$$

Пучок символов $\mathscr{H}_{P}(x, \xi, h)$ является многочленом по совокупности переменных $(\xi, h)$ с бесконечно дифференцируемьми по $x$ коэффициентами и $p_{m}(x, \xi)=\mathscr{H}_{P}(x, \xi, 0)$.

В силу теоремы о неявной функции для любой точки $\left(x^{0}, \eta^{\prime 0}, \xi^{0}, 0\right) \in \Omega \times \mathbb{R}_{\eta^{\prime}}^{n_{1}} \times$ $\mathbb{R}_{\xi}^{n} \times \mathbb{R}_{h}$, где $n_{1}$ - число переменных $\eta^{\prime}$ и векторы $\left(\eta^{\prime 0}, 0\right)$ и $\left(\xi^{\prime 0}, \xi^{\prime \prime 0}\right)$ не коллинеарны, найдется окрестность $W$, в которой уравнение $\mathscr{H}_{P}\left(x, \xi^{\prime}+z \eta^{\prime}, \xi^{\prime \prime}, h\right)=0$ относительно $z \in \mathbb{C}$ имеет $m / \mu$ простых корней $z_{j}\left(x, \eta^{\prime}, \xi, h\right)$.

Предположим, что

II) каж дьй корень $z_{j}\left(x, \eta^{\prime}, \xi, h\right)$ удовлетворяет одному из условий:

a) $\operatorname{Im} z_{j}\left(x^{0}, \eta^{\prime 0}, \xi^{0}, 0\right) \neq 0$

b) $\operatorname{Im} z_{j}\left(x, \eta^{\prime}, \xi, h\right) \equiv 0$ в некоторой окрестности точки $\left(x^{0}, \eta^{\prime 0}, \xi^{0}, 0\right)$. 
Пусть $G$ - ограниченное открытое множество в $\mathbb{R}^{n}$. Говорят, что комплекснозначная (вешественнозначная) функция $f(x, u)$, определенная для почти всех $x \in G$ и всех $u \in \mathbb{C}^{N}$ (соответственно $\left.\mathbb{R}^{N}\right)$, обладает свойством Каратеодори, если для почти всех $x \in G$ она непрерьвна по $u$ и для всех $u$ измерима по $x$. Ниже, чтобы не дублировать формулировки, будем рассматривать случай $u \in \mathbb{C}^{N}$. Принадлежность функции к классу Каратеодори эквивалентна тому, что для любого числа $\varepsilon>0$ существует замкнутое множество $F_{\varepsilon} \subset G$, мера которого больше mes $G-\varepsilon$, такое, что на произведении $F_{\varepsilon} \times \mathbb{C}^{N}$ функция $f(x, u)$ непрерьвна по совокупности всех аргументов [1, теорема 18.2]. Если функция $f(x, u)$ обладает свойством Каратеодори, то для любых измеримых на $G$ функций $\left(u_{1}(x), u_{2}(x), \ldots, u_{N}(x)\right)=u(x)$ сложная функция $f(x, u(x))$ будет измеримой на $G[1$, теорема 18.3]. Для неограниченного открытого множества $\Omega$ будем говорить, что функция $f(x, u)$ обладает свойством Каратеодори, если она обладает этим свойством на любом множестве $G \times \mathbb{C}^{N}$, где $G$ - произвольное ограниченное открытоеподмножество множества $\Omega$.

ОПРЕДЕЛЕНИЕ 1. Скажем, что функция $f(x, u)$ nринадлежит классу $C L_{p}\left(\Omega \times \mathbb{C}^{N}\right)$, $p \geqslant 1$ (кратко $\left.C L_{p}\right)$, если

1) $f(x, u)$ обладает свойством Каратеодори;

2) $f(x, 0) \in L_{p}(\Omega)$ и для любого компакта $K \subset \mathbb{C}^{N}$ найдется константа $C=C(K)$, с которой для всех $u^{1}$ и $u^{2} \in K$ и почти всех $x \in \Omega$ выполняется липшицева оценка

$$
\left|f\left(x, u^{1}\right)-f\left(x, u^{2}\right)\right| \leqslant C\left|u^{1}-u^{2}\right|
$$

Будем говорить, что функция $f(x, u)$ принадлежсит классу $C L_{p, \mathrm{loc}}$, если для любой функции $\varphi(x) \in C_{0}^{\infty}(\Omega)$ произведение $\varphi(x) f(x, u)$ принадлежит классу $C L_{p}$. Если $f(x, u) \in C L_{p, \mathrm{loc}}$, то для любого фиксированного $u$ справедливо включение $f(x, u) \in$ $L_{p, \text { lос }}(\Omega)$. Более того, из $(2)$ следует, что для любых компактов $K_{1} \subset \Omega$ и $K_{2} \subset \mathbb{C}^{N}$ с некоторой константой $C=C\left(K_{1}, K_{2}\right)$ для почти всех $x \in K_{1}$ и всех $u \in K_{2}$ вьполняется оценка

$$
|f(x, u)| \leqslant|f(x, 0)|+C .
$$

Пусть $J$ - подмножество множества мультииндексов, удовлетворяющих неравенству $\langle\varrho, \alpha\rangle \leqslant m-\mu$, и $N(J)-$ их число. Если для каждого $\alpha \in J$ производная $\partial^{\alpha} u(x)$ обобщенной функции $u(x)$ принадлежит пространству $L_{\infty, \operatorname{loc}}(\Omega)$, то векторнозначная функция $\partial_{J} u(x)=\left(\partial^{\alpha} u(x)\right)_{\alpha \in J}$ принадлежит декартовому произведению $N(J)$ экземпляров пространства $L_{\infty, \text { loc }}(\Omega)$. Теперь если $f\left(x, u_{J}\right) \in C L_{p, \text { loc }}$, где $u_{J}=\left(u_{\alpha}\right)_{\alpha \in J}$, то из $(3)$ следует, что сложная функция $f\left(x, \partial_{J} u(x)\right)$ принадлежит пространству $L_{p, \text { loc }}(\Omega)$. Если $u^{1}(x)$ и $u^{2}(x)$ - две функции, обладающие указанньми свойствами гладкости, то для любого компакта $K_{1} \subset \Omega$ найдется компакт $K_{2} \subset \mathbb{C}^{N(J)}$ такой, что для почти всех $x \in K_{1}$ справедливо включение $\partial_{J} u^{j}(x) \in K_{2}, j=1,2$. Из неравенства (2) вытекает, что найдется константа $C$, с которой для почти всех $x \in K_{1}$ выполняется оценка

$$
\left|f\left(x, \partial_{J} u^{1}(x)\right)-f\left(x, \partial_{J} u^{2}(x)\right)\right| \leqslant C \sum_{\alpha \in J}\left|\partial^{\alpha}\left(u^{1}(x)-u^{2}(x)\right)\right|
$$


ОпРЕДЕЛЕниЕ 2. Класс обобщенных функций, определенных на открытом множестве $\Omega \subseteq \mathbb{R}^{n}$, будем называть $x^{\prime}$-квазианалитическим, если из совпадения двух функций класса в некоторой окрестности произвольной точки $x^{0}=\left(x^{\prime 0}, x^{\prime \prime 0}\right) \in \Omega$ следует их совпадение в окрестности связной компоненты $\mathscr{F}{ }_{x^{0}}(\Omega)$ слоя $\left\{x \in \Omega \mid x^{\prime \prime}=x^{\prime \prime 0}\right\}$, содержащей эту точку $x^{0}$.

Квазианалитические классы функций одной переменной исследовались многими авторами. Карлеманом [2] найдены необходимые и достаточные условия квазианалитичности (по Адамару) классов бесконечно дифференцируемых функций одной вещественной переменной. В дальнейшем эти условия видоизменялись. Современный вариант (теорема Данжуа-Карлемана) можно найти в монографии Хёрмандера [3]. Бернштейн [4], используя определение квазианалитичности, близкое к приведенному выше, получил необходимые и достаточные условия квазианалитичности классов функций одной вещественной переменной, связывающие квазианалитичность с приближениями функций многочленами. В многомерном случае квазианалитичность классов функций, образованных решениями дифференциальных уравнений с частными производньми, тесно связана с однозначностью продолжения решений. В изотропном случае подобные результаты можно получить из теорем кальдероновского типа (см., например, [3, теоремы 17.2.1, 28.1.1]). В анизотропном случае Ландисом и Олейник [5] доказана квазианалитичность классов, образованных сужениями решений ряда линейных однородных параболических уравнений на связную компоненту характеристической гиперплоскости.

Скажем, что обобщенная функция $u(x)$ принадлежит пространству $H_{w, \text { loc }}^{m-\mu}(\Omega)$, если для любого мультииндекса $\alpha \in\left(\mathbb{Z}_{+}\right)^{n}$, взвешенный порядок которого не превосходит $m-\mu$, производные $\partial^{\alpha} u(x)$ принадлежат пространству $L_{2, l o c}(\Omega)$. Будем говорить, что обобщенная функция $u(x)$ принадлежит пространству $L_{\infty, J, l o c}(\Omega)$, если $\partial^{\alpha} u(x) \in$ $L_{\infty, \text { loc }}(\Omega)$ при $\alpha \in J$.

ТЕОремА 1. Предположим, что оператор $P(x, D)$ удовлетворяет условиям I) $u$ II) $u f\left(x, u_{J}\right) \in C L_{2, \operatorname{loc}}\left(\Omega \times \mathbb{C}^{N(J)}\right)$. Тогда мнохсество решений $u(x) \in H_{w, l o c}^{m-\mu}(\Omega) \cap$ $L_{\infty, J, \text { lос }}(\Omega)$ уравнения

$$
P(x, D) u=f\left(x, \partial_{J} u(x)\right)
$$

образует $x^{\prime}$-квазианалитический класс.

ДокАЗАТЕЛЬСТВо. Положим

$$
P_{1}(x, D)=\sum_{m-\mu<\langle\varrho, \alpha\rangle \leqslant m} a_{\alpha}(x) D^{\alpha}
$$

Тогда $\mathscr{H}_{P_{1}}(x, \xi, h)=\mathscr{H}_{P}(x, \xi, h)$. В силу $\xi^{\prime}$-квазиэллиптичности оператора $P(x, D)$ и условий I) и II) множество $\left\{(x, \xi, h) \in \Omega \times\left(\mathbb{R}^{n} \backslash\{0\}\right) \mid \mathscr{H}_{P_{1}}(x, \xi, h)=0\right\}$ в окрестности каждой принадлежащей ему точки $\left(x^{0}, \xi^{0}, 0\right)$ является $C^{\infty}$-гиперповерхностью. Вследствие условия I) множество $\Xi$, состоящее из точек $\left(x, \eta^{\prime}\right) \in \Omega \times\left(\mathbb{R}^{n_{1}} \backslash\{0\}\right)$, для которых найдется вектор $\xi \in \mathbb{R}^{n}$ такой, что полином $p_{m}\left(x, \xi^{\prime}+z \eta^{\prime}, \xi^{\prime \prime}\right)$ по $z \in \mathbb{C}$ имеет по крайней мере один корень $z^{0}$ кратности вьше единицы и $\xi+z^{0} \eta \neq 0$, пусто. Пусть $x^{*}$ точка нехарактеристической $C^{1}$-поверхности $\{x \in U \mid \varphi(x)=0\}$, где $U \subset \Omega$-открытое 
множество, $\varphi(x)$ - вешественная $C^{1}$-функция и $\varphi_{x} \neq 0$ на $U$. Тогда в силу теоремы 1.5 из [6] если $u \in H_{w}^{m-\mu}(U), P_{1}(x, D) u \in L_{2}(U), u=0$ в $\{x \in U \mid \varphi(x)<0\}$ и для любого компактного подмножества $K \subset U$ с некоторой константой $C(K)$ для почти всех $x \in K$ справедливо неравенство

$$
\left|P_{1}(x, D) u\right| \leqslant C(K) \sum_{\langle\varrho, \alpha\rangle \leqslant m-\mu}\left|D^{\alpha} u\right|,
$$

то $u=0$ в некоторой окрестности точки $x^{*}$. Если $u_{1}(x)$ и $u_{2}(x)$ - два решения $(5)$, указанного в теореме класса гладкости, то для любого открытого множества $U$, замыкание которого есть компакт, содержашийся в $\Omega$, разность $u=u_{1}-u_{2} \in H_{w}^{m-\mu}(U)$ в силу (4) удовлетворяет неравенству $(6)$ и $P_{1}(x, D) u \in L_{2}(U)$.

Предположим, что утверждение теоремы не верно́. Тогда найдутся два решения $u_{1}(x)$ и $u_{2}(x)$ и связная компонента $\mathscr{F}_{x^{0}}(\Omega)$ слоя $\left\{x \in \Omega \mid x^{\prime \prime}=x^{\prime \prime 0}\right\}$, содержащая две точки, одна из которых принадлежит носителю разности $u=u_{1}-u_{2}$, а другая нет. Повторяя рассуждения, приведенные в [6] при доказательстве теоремы 4.4, можно построить эллипсоид $\mathscr{E}$, определенный уравнением $\varphi(x)=0$, так, что во внутренней области, ограниченной $\mathscr{E}$, выполняется равенство $u=0$, а на самом эллипсоиде найдется нехарактеристическая точка $x^{*}$, принадлежащая носителю $u$. Тогда, как отмечалось выше, разность $u$ должна быть равной нулю в некоторой окрестности точки $x^{*}$. Полученное противоречие доказьвает теорему.

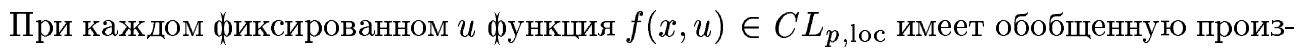
водную $\partial_{x}^{\alpha} f(x, u) \in \mathscr{D}^{\prime}(\Omega)$. Будем говорить, что $\partial_{x}^{\alpha} f(x, u) \in C L_{p, \text { loc }}$, если существует

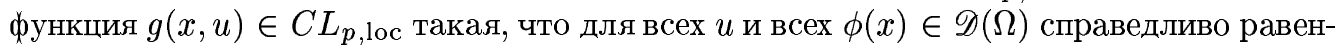
ство

$$
(-1)^{|\alpha|} \int_{\Omega} f(x, u) \partial_{x}^{\alpha} \phi(x) d x=\int_{\Omega} g(x, u) \partial_{x}^{\alpha} \phi(x) d x .
$$

Будем говорить, что функция $f(x, u) \in C L_{p, \text { loc }}\left(\Omega \times \mathbb{R}^{N}\right)$ имеет производную $\partial_{u_{j}} f(x, u)$, принадлежащую классу $C L_{p, \operatorname{loc}}\left(\Omega \times \mathbb{R}^{N}\right)$, если для почти всех $x \in \Omega$ она имеет непрерьвную по $u$ производную $\partial_{u_{j}} f(x, u)$, принадлежащую классу $C L_{p, \text { loc }}\left(\Omega \times \mathbb{R}^{N}\right)$. Аналогично, в комплексном случае для $u \in \mathbb{C}^{N}$ определяются производные $\partial_{u_{j}} f(x, u)$ и $\partial_{\bar{u}_{j}} f(x, u)$. Поскольку $\mathbb{C}^{N} \cong \mathbb{R}^{2 N}$, то правило дифференцирования сложной функции достаточно сформулировать для случая вещественных $u$.

Теорема 2. Предположим, что $u(x) \in L_{\infty, \mathrm{loc}}(\Omega) \otimes \mathbb{R}^{N}, f(x, u) u \partial_{u_{l}} f(x, u) \in$ $C L_{p, \text { lос }}\left(\Omega \times \mathbb{R}^{N}\right)$ при всех $l=1,2, \ldots, N, p \geqslant 1$, и для некоторого $j$ выполнено $\partial_{x_{j}} u(x) \in L_{\infty, \operatorname{loc}}(\Omega) \otimes \mathbb{R}^{N}$ u $\partial_{x_{j}} f(x, u) \in C L_{p, \operatorname{loc}}\left(\Omega \times \mathbb{R}^{N}\right)$. Тогда сложсная функиия $f(x, u(x))$ принадлехст пространству $L_{p, \operatorname{loc}}(\Omega)$ и имеет производную по Соболеву

$$
\partial_{x_{j}} f(x, u(x))=\left(\partial_{x_{j}} f\right)(x, u(x))+\sum_{l=1}^{N}\left(\partial_{u_{l}} f\right)(x, u(x)) \partial_{x_{j}} u_{l}(x),
$$

принадлежсащую пространству $L_{p, \operatorname{loc}}(\Omega)$.

Отметим, чтопри $f(x, u), \partial_{x_{j}} f(x, u) \in C L_{p, l o c}\left(\Omega \times \mathbb{R}^{N}\right)$ и $\partial_{u_{l}} f(x, u) \in C L_{\infty, \operatorname{loc}}\left(\Omega \times \mathbb{R}^{N}\right)$ правая часть равенства (7) порождается подстановкой в функцию

$$
F\left(x, u, u^{\prime}\right)=\left(\partial_{x_{j}} f\right)(x, u)+\sum_{l=1}^{N}\left(\partial_{u_{l}} f\right)(x, u) u_{l}^{\prime}
$$


принадлежащую классу $C L_{p, \operatorname{loc}}\left(\Omega \times \mathbb{R}^{2 N}\right)$, компонент векторной функции $u(x)$ и ее производных по $x_{j}$.

Следующий результат о распространении локальной инвариантности решений уравнений относительно сдвигов является прямым следствием теорем 1 и 2.

Теорема 3. Дополнительно к условиям теоремы 1 предположим, что

1) для некоторого $ј$ коэффициенты уравнения (5) и его правая часть не зависят от переменной $x_{j}$;

2) для любого мультииндекса $\beta \in J$ справедливо включение $\partial_{u_{\beta}} f(x, u) \in C L_{\infty, \mathrm{loc}}$ ( в случае комплекснозначных решений, кроме того, $\left.\partial_{\bar{u}_{\beta}} f(x, u) \in C L_{\infty, \mathrm{loc}}\right)$.

Предположим, что обобщенная функиия $u(x) \in L_{\infty, J, l o c}(\Omega)$ является решением уравнения (5), $\partial_{x_{j}} u(x) \in H_{w, \operatorname{loc}}^{m-\mu}(\Omega)$ и в окрестности точки $\left(x^{\prime 0}, x^{\prime \prime 0}\right)$ функиия $u(x)$ не зависит от $x_{j}$. Тогда $u(x)$ не зависит от $x_{j}$ в некоторой окрестности множества $\mathscr{F}_{x^{0}}(\Omega)$.

ДокАЗАТЕЛьство. Для определенности проведем доказательство в случае вещественнозначных решений. В силу теоремы 2 обобщенная функция $w(x)=\partial_{x_{j}} u(x)$ удовлетворяет линейному дифференциальному уравнению

$$
P(x, D) w=\sum_{\beta \in J} f_{u_{\beta}}\left(x, \partial_{J} u(x)\right) \partial_{x}^{\beta} w(x)
$$

частным решением которого является нулевое решение. Поскольку коэффициенты данного уравнения $f_{u_{\beta}}\left(x, \partial_{J} u(x)\right) \in L_{\infty, \text { loc }}(\Omega)$ и $\langle\varrho, \beta\rangle \leqslant m-\mu$, то в силу теоремы 1 из равенства $w(x)=0$ в окрестности точки $\left(x^{\prime 0}, x^{\prime \prime}\right)$ следует доказьваемое утверждение. В случае комплекснозначных решений правая часть полученного уравнения будет линейной функцией относительно функции $w(x)$, ее комлексно-сопряженной $\bar{w}(x)$ и их производных взвешенного порядка, не превосходящего $m-\mu$, с переменными коэфқиццентами класса $L_{\infty, \text { loc }}(\Omega)$. Это не мешает применению теоремы 1 .

\section{2. Уравнения распространения нелинейных волн.}

Уравнения типа Колмогорова-Петровского-Пискунова. Пусть $\Omega$ - открытое множество в $\mathbb{R}^{n+1}, A(t, x)=\left(a_{l, j}(t, x)\right)$ - симметричная $(n \times n)$-матрища с бесконечно дифференцируемьми коэффициентами.

TЕОрема 4. Предположим, что $f(t, x, v) \in C L_{2}\left(\Omega \times \mathbb{C}^{n+1}\right)$ и в любой точке $(t, x) \in \Omega \subseteq \mathbb{R}^{n+1}$ из равенства $\operatorname{Re}\langle A(t, x) \xi, \xi\rangle=0$ и включения $\xi \in \mathbb{R}^{n}$ следует, что $\xi=0$. Тогда множество решений $u(t, x) \in L_{\infty, \mathrm{loc}}(\Omega)$ уравнения

$$
\left(\frac{\partial}{\partial t}-\sum_{j, l=1}^{n} a_{j, l}(t, x) \frac{\partial^{2}}{\partial x_{j} \partial x_{l}}\right) u=f\left(t, x, u, u_{x_{1}}, \ldots, u_{x_{n}}\right)
$$

производные $u_{x_{j}}, j=1, \ldots, n$, которых принадлежсат $L_{\infty, \mathrm{loc}}(\Omega)$, образует $x$-квазианалитический класс. 
ДоКАЗАТЕЛЬСтво. Введем взвешивание производных, присвоив дифференцированию по $t$ вес 2 , а дифференцированиям по $x_{j}$ вес $1, j=1, \ldots, n$. Тогда $\mu=1$ и для линейного оператора $P$, определяющего левую часть уравнения $(8)$, пучок старших символов совпадает со взвешенным старшим символом: $\mathscr{H}_{P}=p_{2}(t, x, \tau, \xi)=i \tau+\langle A(t, x) \xi, \xi\rangle$, где $(\tau, \xi)$ - двойственные переменные к $(t, x)$ соответственно. Оператор $P$ будет $\xi$-квазиэллиптическим. Покажем, что для него вьполняются условия I) и II) из п. 1. В каждой точке $(t, x) \in \Omega$ для любой пары неколлинеарных векторов $(\tau, \xi)$ и $(0, \eta)$ оба корня квадратного относительно $z \in \mathbb{C}$ уравнения $p_{2}(t, x, \tau, \xi+z \eta)=0$ не являются вещественными. Поэтому достаточно показать, что они различны. При $n=1$ это очевидно. При $n>1$ доказательство аналогично доказательству леммы 17.2.5 из [3]. В самом деле, множество $\mathbb{R}^{n+1} \backslash\{\zeta=\lambda(0, \eta) \mid \lambda \in \mathbb{R}\}$ связно. Поскольку вещественных корней нет, то число корней с положительной мнимой частью не зависит от выбора вектора $(\tau, \xi)$, неколлинеарного вектору $(0, \eta)$. Так как $p_{2}(t, x, \tau,-\xi)=p_{2}(t, x, \tau, \xi)$, то каждому корню с положительной мнимой частью в точке $(t, x, \tau, \xi)$ соответствует корень с отрицательной мнимой частью в точке $(t, x, \tau,-\xi)$. Следовательно, число корней с положительной мнимой частью равно числу корней с отрицательной мнимой частью. Применение теоремы 1 завершает доказательство.

Нелинейное уравнение Шрёдингера. Пусть $\Omega$ - открытое множество в $\mathbb{R}^{2}$.

ТЕОрема 5. Предположим, что $f(t, x, v) \in C L_{2}\left(\Omega \times \mathbb{C}^{2}\right)$ и вещественнозначная функиия $a(t, x) \in C^{\infty}(\Omega)$ отлична от нуля во всех точках $(t, x) \in \Omega$. Тогда множество решений $u(t, x) \in L_{\infty, \mathrm{loc}}(\Omega)$ уравнения

$$
\left(i \frac{\partial}{\partial t}-a(t, x) \frac{\partial^{2}}{\partial x^{2}}\right) u=f\left(t, x, u, u_{x}\right),
$$

производные $u_{x}$ которых принадлежст $L_{\infty, \operatorname{loc}}(\Omega)$, образует $x$-квазианалитический класс.

ДокАЗАТЕЛЬСтво. Взвешивание производных введем, присвоив дифференцированию по $t$ вес 2 , а дифференцированию по $x$ вес 1 . Тогда $\mu=1$. Для линейного оператора $P$ из левой части уравнения (9) пучок старших символов совпадает со взвешенньп старшим символом: $\mathscr{H}_{P}=p_{2}(t, x, \tau, \xi)=\tau-a(t, x) \xi^{2}$, где $(\tau, \xi)$ - двойственные переменныек $(t, x)$ соответственно. Оператор $P$ является $\xi$-квазиэллиптическим. Покажем, что он удовлетворяет условиям I) и II) из п. 1. Пусть $\eta^{0} \neq 0$ и $\tau^{0} \neq 0$. Тогда в любой точке $\left(t^{0}, x^{0}\right)$ относительно $z \in \mathbb{C}$ уравнение $\tau^{0}-a\left(t^{0}, x^{0}\right)\left(\eta^{0}\right)^{2} z^{2}=0$ имеет два различных корня. Кроме того, если $\tau^{0}<0$, то оба корня чисто мнимые и для корней уравнения выполнена часть а) условия II). Если $\tau^{0}>0$, то для всех $\left(t, x, \eta, \tau, \xi^{\prime \prime}\right)$ из некоторой окрестности $W$ точки $\left(t^{0}, x^{0}, \eta^{0}, \tau^{0}, 0\right)$ уравнение $\tau-a(t, x)\left(\xi^{\prime \prime}+z \eta\right)^{2}=0$ относительно $z \in \mathbb{C}$ имеет два различных вещественных корня. Таким образом, в этом случае корни уравнения удовлетворяют части b) условия II). Применяя теорему 1 , завершаем доказательство.

Уравнения типа Кортевега-де Фриза. Пусть $\Omega$-открытое множество в $\mathbb{R}^{2}$.

ТеОрема 6. Предположим, что $f(t, x, v) \in C L_{2}\left(\Omega \times \mathbb{R}^{2}\right)$ и вещественнозначная функция $a(t, x) \in C^{\infty}(\Omega)$ отлична от нуля во всех точках $\Omega$. Тогда мнохество вещ,ественнозначных решений $u(t, x) \in L_{\infty, \mathrm{loc}}(\Omega)$ уравнения

$$
\left(\frac{\partial}{\partial t}+a(t, x) \frac{\partial^{3}}{\partial x^{3}}\right) u=f\left(t, x, u, u_{x}\right),
$$


для производных которых выполняются включения $u_{x} \in L_{\infty, \operatorname{loc}}(\Omega)$ u $u_{x x} \in L_{2, \operatorname{loc}}(\Omega)$, образует х-квазианалитический класс.

ДокАЗАТЕЛЬСтво. Введем взвешивание производных, присвоив дифференцированию по $t$ вес 3 , а дифференцированию по $x$ вес 1 . Тогда $\mu=1$. Пучок старших символов оператора $P$ из левой части уравнения (10) совпадает со взвешенным старшим символом: $\mathscr{H}_{P}=p_{2}(t, x, \tau, \xi)=i\left(\tau-a(t, x) \xi^{3}\right)$, где $(\tau, \xi)$ - двойственные переменные к $(t, x)$ соответственно. Оператор $P$ является $\xi$-квазиэллиптическим. Покажем, что он удовлетворяет условиям I) и II) из п. 1. Пусть $\eta^{0} \neq 0$ и $\tau^{0} \neq 0$. Тогда в любой точке $\left(t^{0}, x^{0}\right)$ относительно $z \in \mathbb{C}$ уравнение $\tau^{0}+a\left(t^{0}, x^{0}\right)\left(\eta^{0}\right)^{3} z^{3}=0$ имеет три различных корня. Очевидно, один из корней удовлетворяет части b) условия II), а два оставшихся - пункту а) условия II). Применение вешественного варианта теоремы 1 завершает доказательство теоремы.

Уравнения типа Кадомщева-Петвиашвили. Пусть $\Omega$ - открытое множество в $\mathbb{R}^{3}$.

Теорема 7. Предположим, что $f(t, x, v) \in C L_{2}\left(\Omega \times \mathbb{R}^{3}\right)$. Тогда множество вешественнозначных решений $u(t, x) \in L_{\infty, \mathrm{loc}}(\Omega)$ уравнения

$$
\left(\frac{\partial^{4}}{\partial x_{1}^{4}}+3 \frac{\partial^{2}}{\partial x_{2}^{2}}+\frac{\partial^{2}}{\partial x_{1} \partial x_{3}}\right) u=f\left(t, x, u, u_{x_{1}}, u_{x_{2}}, u_{x_{1} x_{1}}\right)
$$

для производных которых выполняются включения $u_{x_{1}}, u_{x_{1}}, u_{x_{1} x_{1}} \in L_{\infty, \text { осс }}(\Omega) u$ $u_{x_{3}}, u_{x_{1} x_{2}}, u_{x_{1} x_{1} x_{1}} \in L_{2, \text { loc }}(\Omega)$, образует $x_{1}$-квазианалитический класс.

ДокАЗАТЕЛьСТво. Введем взвешивание производных: $\varrho_{1}=1, \varrho_{2}=2$ и $\varrho_{3}=3$. Тогда $\mu=1$. Для линейного оператора $P$ в левой части уравнения $(11)$ пучок старших символов совпадает со взвешенным старшим символом: $\mathscr{H}_{P}=p_{4}(x, \xi)=\xi_{1}^{4}-3 \xi_{2}^{2}-\xi_{1} \xi_{3}$. Оператор $P$ является $\xi_{1}$-квазиэллиптическим. Можно показать, что при $\eta_{1}^{0} \neq 0$ и $\left(0, \xi_{2}^{0}, \xi_{3}^{0}\right) \neq 0$ многочлен $p\left(z, \eta^{0}\right)=\left(\eta_{1}^{0}\right)^{4} z^{4}-3\left(\xi_{2}^{0}\right)^{2}-\eta_{1}^{0} \xi_{3}^{0} z$ не имеет кратных корней. Коэффициенты многочлена $p(z, \eta)$ являются вещественнозначньпии функциями переменных $\eta \in \mathbb{R}^{3}$. Поэтому из вещественного варианта теоремы о неявной функции следует, что каждьй вещественньй корень многочлена $p\left(z, \eta^{0}\right)$ удовлетворяет части b) условия II). Таким образом, оператор $P$ удовлетворяет условиям теоремы 1 , применение которой завершает доказательство.

Уравнения типа Буссинеска. Пусть $\Omega$ - открытое множество в $\mathbb{R}^{2}$.

Теорема 8. Предположим, ито $f(t, x, v) \in C L_{2}\left(\Omega \times \mathbb{R}^{3}\right) u a(t, x) \in C^{\infty}(\Omega)-$ вещественнозначная полохительная функиия. Тогда мнохество вещ, ественнозначных решений $u(t, x) \in L_{\infty, \mathrm{loc}}(\Omega)$ уравнения

$$
\left(\frac{\partial^{2}}{\partial t^{2}}+a(t, x) \frac{\partial^{4}}{\partial x^{4}}\right) u=f\left(t, x, u, u_{x}, u_{x x}\right)
$$

для производных которых выполняются включения $u_{x}, u_{x x} \in L_{\infty, \operatorname{loc}}(\Omega) u u_{x x x} \in$ $L_{2, \text { loc }}(\Omega)$, образует $x$-квазианалитический класс. 
ДоКАЗАТЕЛЬСтво. Введем взвешивание производных, присвоив дифференцированию по $t$ вес 2 , а дифференцированию по $x$ вес 1 . Тогда $\mu=1$. Пучок старших символов линейного оператора $P$ из левой части уравнения (12) совпадает со взвешенньм старшим символом: $\mathscr{H}_{P}=p_{4}(t, x, \tau, \xi)=-\tau^{2}-a(t, x) \xi^{4}$, где $(\tau, \xi)$ - двойственные переменные к $(t, x)$ соответственно. Оператор $P$ является $\xi$-квазиэллиптическим. Легко проверить, что при $\eta^{0} \neq 0$ и $\tau^{0} \neq 0$ в любой точке $\left(t^{0}, x^{0}\right)$ многочлен $-\left(\tau^{0}\right)^{2}-a\left(t^{0}, x^{0}\right)\left(\eta^{0}\right)^{4} z^{4}$ относительно $z \in \mathbb{C}$ не имеет ни кратных, ни вещественных корней. Поэтому для оператора $P$ вьполняются условия I) и II) из п. 1. Применение вещественного варианта теоремы 1 завершает доказательство.

ЗАмЕчАНИЕ 1. После очевидных уточнений формулировок теоремы 6-8 справедливы для комплекснозначных решений уравнений, а теорема 4 - для вещественнозначных.

ЗАмЕчАниЕ 2. Если нелинейные члены уравнений (8)-(12) не зависят от производных решений или зависят от меньшего их числа, то теоремы верны при более слабых требованиях на априорную гладкость решений. Достаточно предполагать, чтобы “исключенные" производные принадлежали пространству $L_{2, l o c}(\Omega)$. В том случае, когда нелинейные члены уравнений (10)-(12) зависят от большего числа производных решения младшего взвешенного порядка, теоремы верны, если усилить требования на априорную гладкость решений. Достаточно заменить условие принадлежности соответствующих производных пространству $L_{2, l o c}(\Omega)$ на условие их принадлежности пространству $L_{\infty, \text { loc }}(\Omega)$.

ЗАмЕчАнИЕ 3. Из теорем 4-6 и 8 вытекает следующий результат о “стационарном" решении. Предположим, что коэффициенты уравнений не зависят от переменной $t$. Пусть $\Omega=\left(t_{1}, t_{2}\right) \times G$ и $u_{0}(x)$ есть стационарное решение уравнения, обладающее указанными в теореме 4 ( 5,6 и 8 соответственно) свойствами регулярности. Тогда имеет место

СлЕдСТВИЕ. Если $u(t, x)$ принадлежсит классу решений теоремы $4(5,6$ и 8 соответственно) $и$ и $(t, x)=u_{0}(x)$ в окрестности точки $\left(t^{0}, x^{0}\right)$, то $u(t, x)=u_{0}(x)$ в некоторой окрестности слоя $\left\{t^{0}\right\} \times G$.

В том случае, когда функция $f(t, x, v)$, порождающая правую часть соответствующего уравнения, обрашается в нуль при $v=0$, утверждение следствия справедливо для $u_{0}(x)=0$ без предположения о независимости коэффициентов от переменной $t$.

Дополнение. В дополнении обосновывается правило дифференцирования сложной функции $f(x, u(x))$, где $f(x, u) \in C L_{p, \text { loc }}\left(\Omega \times \mathbb{C}^{N}\right)$ (или $\left.C L_{p, \text { loc }}\left(\Omega \times \mathbb{R}^{N}\right)\right), u(x) \in$ $L_{\infty, \text { lос }}(\Omega) \otimes \mathbb{C}^{N}$ (или $L_{\infty, \text { lос }}(\Omega) \otimes \mathbb{R}^{N}$ соответственно) и имеет производную по переменной $x_{j}$ того же класса гладкости. Поскольку $\mathbb{C}^{N} \cong \mathbb{R}^{2 N}$, то достаточно рассмотреть один случай.

Предположим вначале, что $\Omega$ - ограниченное открытоемножествов в $\mathbb{R}^{n}$. Для $f(x, u) \in$ $C L_{p}\left(\Omega \times \mathbb{R}^{N}\right)$ обозначим через $f_{h}(x, u)$ среднюю по $x$ функцию

$$
f_{h}(x, u)=\int_{\Omega} w_{h}(x-y) f(y, u) d y, \quad w_{h}(x)=\frac{1}{h^{n}} w\left(\frac{x}{h}\right),
$$

где $w(x)$ - ядро осреднения, $h$ - положительньй параметр. Будем предполагать, что ядро $w(x)$ - неотрицательная, бесконечно дифференцируемая функция с носителем, содержащимся в единичном шаре, интеграл от которой по $\mathbb{R}^{n}$ равен единице. 
Лемма 1. Если $f(x, u) \in C L_{p}\left(\Omega \times \mathbb{R}^{N}\right)$, mo $f_{h}(x, u) \in C\left(\Omega \times \mathbb{R}^{N}\right)$ для любого $h>0$.

ДокАЗАТЕЛЬСтво. Утверждение леммы очевидным образом выводится из свойства Каратеодори и абсолютной непрерывности интеграла Лебега.

ЛЕмма 2. Пусть $u(x) \in L_{\infty, \operatorname{loc}}(\Omega) \otimes \mathbb{R}^{N} u f(x, u) \in C L_{p}\left(\Omega \times \mathbb{R}^{N}\right), p \geqslant 1$. Тогда $f_{h}(x, u(x)) \rightarrow f(x, u(x))$ в $L_{1, \mathrm{loc}}(\Omega)$ nри $h \rightarrow+0$.

ДокАЗАТЕЛЬСтво. Достаточно показать, что для произвольного шара $K=\{x \mid$ $\left.\left|x-x^{0}\right| \leqslant \mathbb{R}\right\} \subset \Omega$

$$
\lim _{h \rightarrow+0}\left\|f_{h}(x, u(x))-f(x, u(x))\right\|_{L_{1}(K)}=0 .
$$

Выберем число $\delta_{0}>0$ так, чтобы $K_{0}=\left\{x|| x-x^{0} \mid \leqslant \mathbb{R}+\delta_{0}\right\} \subset \Omega$. Поскольку $u(x) \in L_{\infty, \text { lос }}(\Omega) \otimes \mathbb{R}^{N}$, то найдется число $r>0$ такое, что векторная функция $u(x)$ для почти всех $x \in K_{0}$ принимает значения, принадлежашие множеству $B_{r}=\left\{u \in \mathbb{R}^{N} \mid\right.$ $\left.\left|u_{j}\right| \leqslant r, j=1,2, \ldots, N\right\}$.

Пусть $\varepsilon>0$ - произвольное число. Вследствие абсолютной непрерьвности интеграла Лебега и оценки (3) найдется число $\delta_{1}>0$ такое, что для любого множества $K^{\prime} \subset K$, мера которого не превосходит $\delta_{1}$, при $|z| \leqslant \delta_{0}$ справедлива оценка

$$
\int_{K^{\prime}}|f(x-z, u(x))| d x<\frac{\varepsilon}{3} .
$$

Из свойства Каратеодори следует, что найдется замкнутое множество $F \subset \Omega$ с мерой, удовлетворяющей неравенству mes $F>\operatorname{mes} \Omega-\delta_{1} / 4$, такое, что $f(x, u) \in C\left(F \times \mathbb{R}^{N}\right)$. Вследствие равномерной непрерьвности функции $f(x, u)$ на компактном множестве $F \times B_{r}$ найдется число $\delta_{2} \in\left(0, \delta_{0}\right)$ такое, что для всех $x, x-z \in F,|z|<\delta_{2}$ и $u \in B_{r}$ выполняется неравенство

$$
|f(x-z, u)-f(x, u)|<\frac{\varepsilon}{3}(\operatorname{mes} \Omega)^{-1} .
$$

Выберем компактное множество $K_{1} \subset K \cap F$, отстоящее от границы шара $K$ на положительном расстоянии $d$, так, чтобы mes $K_{1}>$ mes $K-\delta_{1} / 2$ и для всех $x \in K_{1}$ и для $j=1,2, \ldots, N$ были справедливы оценки $\left|u_{j}(x)\right| \leqslant r$. Очевидно, $K_{1, z}=\left\{x+z \mid x \in K_{1}\right\}$ $\subset K$ при $|z| \leqslant \delta_{0}^{\prime}=\min \left\{\delta_{0}, d\right\}$. Так как mes $K_{1, z}=\operatorname{mes} K_{1}$, то для меры пересечения $K_{z}^{*}=K_{1} \cap K_{1, z}$ вьполняется неравенство $\operatorname{mes} K_{z}^{*} \geqslant \operatorname{mes} K_{1}-\operatorname{mes}\left(K \backslash K_{1, z}\right)=$ $2 \operatorname{mes} K_{1}-\operatorname{mes} K>\operatorname{mes} K-\delta_{1}$. Пусть $h<\delta_{0}^{\prime}$. Тогда

$$
\begin{aligned}
& \left\|f_{h}(x, u(x))-f(x, u(x))\right\|_{L_{1}(K)} \\
& \quad \leqslant \int_{|z| \leqslant h} w_{h}(z)\left[\left(\int_{K_{z}^{*}}+\int_{K \backslash K_{z}^{*}}\right)|f(x-z, u(x))-f(x, u(x))| d x\right] d z .
\end{aligned}
$$

Внутренний интеграл в правой части последнего неравенства по множеству $K_{z}^{*}$ оценивается через $\varepsilon / 3$ при $|z| \leqslant h$ по построению $K_{z}^{*}$. Поскольку $\operatorname{mes}\left(K \backslash K_{z}^{*}\right) \leqslant \delta_{1}$, то внутренний интеграл по множеству $K \backslash K_{z}^{*}$ оценивается через $2 \varepsilon / 3$. Отсюда следует, что при $h<\delta_{0}^{\prime}$ вьполняется неравенство $\left\|f_{h}(x, u(x))-f(x, u(x))\right\|_{L_{1}(K)}<\varepsilon$. Лемма доказана. 
Пусть $f: \mathbb{R}^{N} \rightarrow \mathscr{D}^{\prime}(\Omega)$. Предположим, что для любой функции $\phi(x) \in \mathscr{D}(\Omega)$ числовая функция $f_{\phi}(u)=\langle f(x, u), \phi(x)\rangle$ имеет в точке $u^{0} \in \mathbb{R}^{N}$ частную производную $\partial_{u_{j}} f_{\phi}\left(u^{0}\right)$. Тогда в силу полноты пространства $\mathscr{D}^{\prime}(\Omega)$ найдется функция $w(x) \in \mathscr{D}^{\prime}(\Omega)$, с которой для всех $\phi(x) \in \mathscr{D}(\Omega)$ вьполняется равенство $\partial_{u_{j}} f_{\phi}\left(u^{0}\right)=\langle w(x), \phi(x)\rangle$. В этом случае будем говорить, что $f(x, u)$ имеет частную производную $\partial_{u_{j}} f\left(x, u^{0}\right)=w(x)$. Из существования производной $\partial_{u_{j}} f\left(x, u^{0}\right)$ и определения операции дифференцирования обобщенной функции следует, что для любого неотрицательного целочисленного мультииндекса $\alpha$ отображение $\partial_{x}^{\alpha} f(x, u): \mathbb{R}^{N} \rightarrow \mathscr{D}^{\prime}(\Omega)$ имеет частную производную $\partial_{u_{j}}\left(\partial_{x}^{\alpha} f\right)\left(x, u^{0}\right)$, причем $\partial_{u_{j}}\left(\partial_{x}^{\alpha} f\right)\left(x, u^{0}\right)=\partial_{x}^{\alpha}\left(\partial_{u_{j}} f\left(x, u^{0}\right)\right)$. Мы говорим, что отображение $f: \mathbb{R}^{N} \rightarrow \mathscr{D}^{\prime}(\Omega)$ принадлежит классу $C L_{p}\left(\Omega \times \mathbb{R}^{N}\right), p \geqslant 1$, если существует функция $g(x, u) \in C L_{p}\left(\Omega \times \mathbb{R}^{N}\right)$ такая, что

$$
f_{\phi}(u)=\int_{\Omega} g(x, u) \phi(x) d x
$$

для всех $\phi(x) \in \mathscr{D}(\Omega)$ и $u \in \mathbb{R}^{N}$. Если $f(x, u) \in C L_{p}\left(\Omega \times \mathbb{R}^{N}\right)$, то $f_{\phi}(u) \in C\left(\mathbb{R}^{N}\right)$. Этот факт доказывается аналогично лемме 1. Из теоремы Лебега о предельном переходе под знаком интеграла выводится следующее утверждение о существовании частных производных.

Лемма 3. Предположими, ито $f(x, u) \in C L_{p}\left(\Omega \times \mathbb{R}^{N}\right)$ и для некоторого $j$, почти всех $x \in \Omega$ и всех $u \in \mathbb{R}^{N}$ существует частная производная $\partial_{u_{j}} f(x, u)$, причем для любого компактного подмножества $K \subset \mathbb{R}^{N}$ найдется неотрицательная суммируемая по $x \in \Omega$ функиия $g_{K}(x)$ такая, что $\left|\partial_{u_{j}} f(x, u)\right| \leqslant g_{K}(x)$ для почти всех $x \in \Omega$ и всех $u \in K$. Тогда для любого $u \in \mathbb{R}^{N}$ функиия $\partial_{u_{j}} f(x, u)$ суммируема по $x \in \Omega$ и для любой функиии $\phi(x) \in \mathscr{D}(\Omega)$ для всех $u \in \mathbb{R}^{N}$ справедливо равенство

$$
\frac{\partial}{\partial u_{j}} \int_{\Omega} f(x, u) \phi(x) d x=\int_{\Omega} \partial_{u_{j}} f(x, u) \phi(x) d x .
$$

Если функция $f(x, u) \in C L_{p}\left(\Omega \times \mathbb{R}^{N}\right)$ для почти всех $x \in \Omega$ имеет непрерывную по $u$ производную $\partial_{u_{j}} f(x, u)$, принадлежащую $C L_{p}\left(\Omega \times \mathbb{R}^{N}\right)$, то существование мажорантных функций вытекает из определения класса $C L_{p}\left(\Omega \times \mathbb{R}^{N}\right)$. В этом случае мы говорим, что функция $f(x, u) \in C L_{p}\left(\Omega \times \mathbb{R}^{N}\right)$ имеет производную $\partial_{u_{j}} f(x, u) \in C L_{p}\left(\Omega \times \mathbb{R}^{N}\right)$.

ЛЕмма 4. Если $f(x, u)$ и $\partial_{u_{j}} f(x, u) \in C L_{p}\left(\Omega \times \mathbb{R}^{N}\right)$, то для любого $h>0$ функиия $f_{h}(x, u)$ непрерывно дифферениируема по $u_{j}$ на множестве $\Omega \times \mathbb{R}^{N}$. Eсли $f(x, u)$ $u \partial_{x_{l}} f(x, u) \in C L_{p}\left(\Omega \times \mathbb{R}^{N}\right)$, то для любого $h>0$ функция $f_{h}(x, u)$ непрерывно дифференчируема по $x_{l}$ на множестве $\Omega_{h} \times \mathbb{R}^{N}$, где $\Omega_{h}=\{x \in \Omega|| x-y \mid>h \forall y \notin \Omega\}$.

ДокАЗАтЕльСтво. Оба утверждения вытекают из леммы 1. При доказательстве второго следует воспользоваться тем, что $\partial_{x_{l}} f_{h}(x, u)=\left(\partial_{x_{l}} f\right)_{h}(x, u)$ на $\Omega_{h} \times \mathbb{R}^{N}$.

ДОКАЗАТЕЛЬСТВО ТЕОРЕМЫ 2. По определению класса $C L_{p}$ функция $f(x, u(x))$ и правая часть равенства $(7)$ принадлежат пространству $L_{p, l o c}(\Omega)$. Поэтому достаточно доказать равенство (7). Поскольку оно носит локальньй характер, то можно считать, что $\Omega$ - ограниченное множество и участвующие в формулировке теоремы функции принадлежат соответствующим пространствам и классам без приставки "lос". С помощью 
лемм 1,2 и 4 доказательство равенства сводится к тому случаю, когда функция $f(x, u)$ непрерьвна в $\Omega \times \mathbb{R}^{N}$ и имеет непрерывные производные $\partial_{x_{j}} f(x, u)$ и $\partial_{u_{l}} f(x, u)$ при $l=1,2, \ldots, N$. Выберем число $r$ так, чтобы для почти всех $x \in \Omega$ вьполнялось неравенство $|u(x)|<r$. Пусть срезающая функция $e_{r}(u) \in \mathscr{D}\left(\mathbb{R}^{N}\right)$ выбрана так, что $e_{r}(u)=1$ в некоторой окрестности шара $\left\{u \in \mathbb{R}^{N}|| u \mid \leqslant r\right\}$. Очевидно, равенство (7) достаточно доказать для функции $f_{r}(x, u)=e_{r}(u) f(x, u)$. Вследствие непрерьвности производных $\partial_{u_{l}} f(x, u)$ для любого открытого множества $\Omega^{\prime}$, замыкание которого есть компакт, содержашийся в $\Omega$, найдется константа $C_{1}$, с которой для для почти всех $x \in \Omega^{\prime}$ и всех $u$ и $v \in \mathbb{R}^{N}$ вьполняется липшицева оценка

$$
\left|f_{r}(x, u)-f_{r}(x, v)\right| \leqslant C_{1}|u-v| .
$$

В силу известных свойств пространств Соболева найдется последовательность функций $\left\{u^{m}(x)\right\}_{m=1}^{\infty} \in C^{\infty}(\Omega) \otimes \mathbb{R}^{N}$ такая, что $u^{m}(x) \rightarrow u(x)$ и $\partial_{x_{j}} u^{m}(x) \rightarrow \partial_{x_{j}} u(x)$ в пространстве $L_{1, \text { lос }}(\Omega) \otimes \mathbb{R}^{N}$ при $m \rightarrow \infty$. Переходя в случае необходимости к подпоследовательности, можно считать, что $u^{m}(x) \rightarrow u(x)$ почти всюду в $\Omega$ при $m \rightarrow \infty$. В силу (13)

$$
\int_{\Omega^{\prime}}\left|f_{r}\left(x, u^{m}(x)\right)-f_{r}(x, u(x))\right| d x \leqslant C_{1} \int_{\Omega^{\prime}}\left|u^{m}(x)-u(x)\right| d x \rightarrow 0
$$

при $m \rightarrow \infty$. Таким образом, $f_{r}\left(x, u^{m}(x)\right) \rightarrow f_{r}(x, u(x))$ в $L_{1, \text { loc }}(\Omega)$ при $m \rightarrow \infty$. Из ограниченности на $\Omega^{\prime} \times \mathbb{R}^{N}$ производных $\partial_{u_{l}} f_{r}(x, u)$ с некоторой константой $C_{2}$ получаем

$$
\begin{aligned}
& \int_{\Omega^{\prime}}\left|\left(\partial_{u_{l}} f_{r}\right)\left(x, u^{m}(x)\right) \partial_{x_{j}} u_{l}^{m}-\left(\partial_{u_{l}} f_{r}\right)(x, u(x)) \partial_{x_{j}} u_{l}\right| d x \\
& \quad \leqslant C_{2} \int_{\Omega^{\prime}}\left|\partial_{x_{j}} u_{l}^{m}-\partial_{x_{j}} u_{l}\right| d x+\int_{\Omega^{\prime}}\left|\left(\partial_{u_{l}} f_{r}\right)\left(x, u^{m}(x)\right)-\left(\partial_{u_{l}} f_{r}\right)(x, u(x))\right|\left|\partial_{x_{j}} u_{l}\right| d x .
\end{aligned}
$$

Первое слагаемое справа стремится к нулю при $m \rightarrow \infty$ в силу выбора последовательности $\left\{u^{m}(x)\right\}_{m=1}^{\infty}$. Второе также стремится к нулю вследствие теоремы Лебега о предельном переходе под знаком интеграла. Теперь равенство (7) следует из известного в анализе правила дифференцирования сложной функции $f_{r}\left(x, u^{m}(x)\right)$. Теорема 2 доказана.

\section{СПИСОК ЦИТИРОВАННОЙ ЛИТЕРАТУРЫ}

[1] Вайнберг М. М. Вариационные методы исследования нелинейных операторов. М.: Гостехиздат, 1956.

[2] Carleman T. Les fonctions quasi-analytiques. Paris, 1926.

[3] Хёрмандер Л. Анализ линейных дифференциальных операторов с частными производными. Т. 1-4. М.: Мир, 1987.

[4] Бернштейн С. Н. Аналитические функции вещественной переменной, их возникновение и пути обобщений // Собрание сочинений. Т. 1. М.: Изд-во АН СССР, 1952. С. 285-320.

[5] Л андис Е. М., Олейник О. А. Обобщенная аналитичность и некоторые связанные с ней свойства решений эллиптических и параболических уравнений // УМН. 1974. Т. 29. № 2. C. $190-206$.

[6] Шананин Н. А. Об однозначном продолжении решений дифференциальных уравнений со взвешенньми производными // Матем. сб. 2000. Т. 191. №3. С. 113-142. 